\title{
REGULARITY OF MEAN CURVATURE FLOW OF GRAPHS ON LIE GROUPS FREE UP TO STEP 2
}

\author{
LUCA CAPOGNA, GIOVANNA CITTI, AND MARIA MANFREDINI
}

\begin{abstract}
We consider (smooth) solutions of the mean curvature flow of graphs over bounded domains in a Lie group free up to step two (and not necessarily nilpotent), endowed with a one parameter family of Riemannian metrics $\sigma_{\epsilon}$ collapsing to a subRiemannian metric $\sigma_{0}$ as $\epsilon \rightarrow 0$. We establish $C^{k, \alpha}$ estimates for this flow, that are uniform as $\epsilon \rightarrow 0$ and as a consequence prove long time existence for the subRiemannian mean curvature flow of the graph. Our proof extend to the setting of every step two Carnot group (not necessarily free) and can be adapted following our previous work in [10] to the total variation flow.
\end{abstract}

\section{INTRODUCTION}

The mean curvature flow is the motion of a surface where each point is moving in the direction of the normal with speed equal to the mean curvature. In the case where the evolution of graphs $S_{t}=\{(x, u(x, t))\} \subset \mathbb{R}^{n} \times \mathbb{R}$ is considered, then, provided enough regularity is assumed, the function $u$ satisfies the equation

$$
\partial_{t} u=\sqrt{1+|\nabla u|^{2}} d i v\left(\frac{\nabla u}{\sqrt{1+|\nabla u|^{2}}}\right) .
$$

Given appropriate boundary/initial conditions, global in time solutions asymptotically converge to minimal graphs.

In this paper we study long time existence of graph solutions of the mean curvature flow in a special class of degenerate Riemannian ambient spaces: The setting of sub-Riemannian manifolds [22], [40]. In particular we will focus on a class of Lie groups endowed with a metric structure $\left(G, \sigma_{0}\right)$ that arises as limit of collapsing left-invariant Riemannian structures $\left(G, \sigma_{\epsilon}\right)$.

Our approach to the existence of global (in time) smooth solutions is based on a Riemannian approximation scheme. We study graph solutions of the mean curvature flow in the Riemannian spaces $\left(G, \sigma_{\epsilon}\right)$ where $G$ is a group and $\sigma_{\epsilon}$ is a family of Riemannian metrics that 'collapse' as $\epsilon \rightarrow 0$ to a sub-Riemannian metric $\sigma_{0}$ in $G$.

Key words and phrases. mean curvature flow, sub-Riemannian geometry, Carnot groups The authors are partially funded by MAnET "Metric Analysis for Emergent Technologies", Marie Curie Initial Training Network, grant agreement n. 607643 (GC and MM) and by the NSF award DMS-1449143 (LC) . 
Our results are analogue to those we proved for the total variation flow proved in [10]. The main difference is that we remove here the assumption that the group $G$ is a Carnot group, i.e. we also consider non-nilpotent groups such as the group of rigid Euclidean motions $\mathcal{R} \mathcal{T}$. In fact the results in the present paper yield, with minor modifications of the proof, the regularity and long time existence of the total variation flow in the same extended class of groups. The main new technical challenge that distinguishes the study of the total variation flow from the mean curvature flow is that the equation studied here is not in divergence form, which makes it necessary to completely change the proof of the $C^{1, \alpha}$ regularity.

1.1. Lie group structure. Let $G$ be an analytic and simply connected Lie group with topological dimension $n$.

A subRiemannian manifold on $G$ is a triplet $\left(G ; \Delta ; \sigma_{0}\right)$ where $\Delta$ denotes a left invariant bracket-generating subbundle of $T M$, and $\sigma_{0}$ is a positive definite smooth, bilinear form on $\Delta$, (see for instance Montgomery [32]). We fix a orthonormal horizontal basis $X_{1}, \ldots, X_{m}$ of $\Delta$. We will say that the group has step 2 if $\left\{X_{i}\right\}_{i=1, \ldots, m} \cup\left\{\left[X_{i}, X_{j}\right]\right\}_{i, j=1, \ldots, m}$ span the whole tangent space at every point. If in addition the vector fields

$$
\left\{X_{i}\right\}_{i=1, \ldots, m} \text { and }\left\{\left[X_{i}, X_{j}\right]\right\}_{i, j=1, \ldots, m}
$$

are linearly independent we say that the group is free up to step 2. We can complete $X_{1}, \ldots, X_{m}$ to a basis $\left(X_{1}, \ldots, X_{n}\right)$ of the Lie algebra $\mathcal{G}$, choosing a basis of the second layer of the tangent space. We denote by $\left(X_{1}, \ldots, X_{n}\right)\left(\operatorname{resp} .\left(X_{1}^{r}, \ldots, X_{n}^{r}\right)\right)$ the left invariant (resp. right invariant) translations of the frames $\left(X_{1}, \ldots, X_{n}\right)$. We will say that the vector fields $X_{1}, \ldots, X_{m}$ have degree 1 and denote $d\left(X_{i}\right)=1$ while their commutators of the form $\left[X_{i}, X_{j}\right]$ have degree 2 . Throughout the paper we will assume for simplicity that the horizontal frame above is self-adjoint, the results continue to hold with minimal modifications of the proofs if this hypothesis is not satisfied.

As prototypes for this class of spaces we highlight the following:

- The standard example for such families is the Heisenberg group $\mathbb{H}^{1}$. This is a Lie group whose underlying manifold is $\mathbb{R}^{3}$ and is endowed with a group law

$$
\left(x_{1}, x_{2}, x_{3}\right)\left(y_{1}, y_{2}, y_{3}\right)=\left(x_{1}+y_{1}, x_{2}+y_{2}, x_{3}+y_{3}-\left(x_{2} y_{1}-x_{1} y_{2}\right)\right) .
$$

With respect to such law one has that the vector fields

$$
X_{1}=\partial_{x_{1}}-x_{2} \partial_{x_{3}} \text { and } X_{2}=\partial_{x_{2}}+x_{1} \partial_{x_{3}}
$$

are left-invariant. Together with their commutator $\left[X_{1}, X_{2}\right]=2 \partial_{x_{3}}$ they yield a basis of $\mathbb{R}^{3}$.

- A second example is given by the classical group of rigid motions of the plane, also known as the roto-translation group $\mathcal{R} \mathcal{T}$. This is a Lie group with underlying manifold $\mathbb{R}^{2} \times S^{1}$ and a group law $\left(x_{1}, x_{2}, \theta_{1}\right)\left(y_{1}, y_{2}, \theta_{2}\right)=\left(x_{1}+y_{1} \cos \theta-y_{2} \sin \theta, x_{2}+y_{1} \sin \theta+\right.$ $\left.y_{2} \cos \theta, \theta_{1}+\theta_{2}\right)$. The horizontal distribution is given by

$$
\Delta=\operatorname{span}\left\{X_{1}, X_{2}\right\} \text {, with } X_{1}=\cos \theta \partial_{x_{1}}+\sin \theta \partial_{x_{2}} \text {, and } X_{2}=\partial_{\theta} .
$$


The subRiemannian metric $\sigma_{0}$ is defined so that $X_{1}$ and $X_{2}$ form a orthonormal basis. Note that $X_{1}, X_{2}$ and $\left[X_{1}, X_{2}\right]=X_{3}=-\sin \theta \partial_{x_{1}}+\cos \theta \partial_{x_{2}}$ span the tangent space at every point.

The assumption that $\Delta$ is bracket generating, allows to use the results from [35], and define a control distance $d_{0}(x, y)$ associated to the distribution $X_{1}, \ldots, X_{m}$, which is called the Carnot-Carathéodory metric (denote by $d_{r, 0}$ the corresponding right invariant distance). We let $\nabla_{0}=\left(X_{1}, \ldots, X_{m}\right)$ denote the horizontal gradient operator. If $\phi \in C^{\infty}(G)$ we set $\nabla_{0} \phi=\sum_{i=1}^{m} X_{i} \phi X_{i}$ and $\left|\nabla_{0} \phi\right|^{2}=\sum_{i=1}^{m}\left(X_{i} \phi\right)^{2}$.

We define a family of left invariant Riemannian metrics $\sigma_{\epsilon}, \epsilon>0$ in $\mathcal{G}$ by requesting that

$$
\left\{X_{1}^{\epsilon}, \ldots, X_{n}^{\epsilon}\right\}:=\left\{X_{1}, \ldots, X_{m}, \epsilon X_{m+1}, \ldots, \epsilon X_{n}\right\}
$$

is an orthonormal frame. We will denote by $d_{\epsilon}$ the corresponding distance functions. Correspondingly we use $\nabla_{\epsilon}$, (resp. $\nabla_{\epsilon}^{r}$ ) to denote the left (resp. right) invariant gradients. In particular, if $\phi \in C^{\infty}(G)$ we set $\nabla_{\epsilon} \phi=\sum_{i=1}^{n} X_{i}^{\epsilon} \phi X_{i}^{\epsilon}$ and $\left|\nabla_{\epsilon} \phi\right|^{2}=\sum_{i=1}^{n}\left(X_{i}^{\epsilon} \phi\right)^{2}$.

Our results rest in a crucial way on the use of the celebrated Rothschild and Stein approximation theorem [38]. Let $X_{1}, \ldots, X_{m}$ be a bracket generating family of smooth vector fields, free up to step 2 , denote by $X_{1}, \ldots, X_{n}$ its completion to a basis of of the tangent bundle $T G$ and set for every $u \in \mathbb{R}^{n}$,

$$
\Phi_{x}(u)=\exp \left(\sum_{i=1}^{n} u_{i} X_{i}\right)(x) .
$$

We will use the following special case of the Rothschild-Stein osculating theorem [38, Theorem 5], to approximate a neighborhood of any point in $G$ with a a neighborhood of the identity in the free group $G_{m, 2}$ with $m$ generators $Y_{1}, \ldots, Y_{m}$ and step 2.

Theorem 1.1. Let $X_{1}, \ldots, X_{m}$ be a family of smooth vector fields in $G$ that are free up to rank 2 at every point, as defined above. Let $G_{m, 2}$ be the free Lie group of step two, with $m$ generators $Y_{1}, \ldots, Y_{m}$ and set $Y_{1}, \ldots, Y_{n}$ to be the basis obtained by the original generators and their commutators. For every $x \in G$ there exists a neighborhood $V$ of $x$ and a neighborhood $U$ of the identity in $G_{m, 2}$ such that:

(a) the map $\Phi_{x}: U \rightarrow V$ is a diffeomorphism onto its image. We will denote by $\Theta_{x}$ its inverse map. Here we have denoted points in $U$ by their coordinates $\left(u_{1}, \ldots, u_{n}\right)$ in the basis $Y_{1}, \ldots, Y_{n}$.

(b) we have

$$
d \Theta_{x}\left(X_{i}\right)=Y_{i}+R_{i}, \quad i=1, \ldots, m
$$

where $R_{i}$ is a vector field of local degree less or equal than zero, depending smoothly on $x$. This means that the operator $R_{i}$ is represented as

$$
R_{i}=\sum_{h=1}^{n} \sigma_{i h}(u) X_{h},
$$


where each $\sigma_{i h}$ has a a Taylor expansion of homogeneous functions of degree larger or equal than $d\left(X_{h}\right)$.

1.2. The mean curvature flow. If a surface $M$ is represented as a 0 -level set of a function $f$, the points where the horizontal gradient of the defining function does not vanish are called non characteristic. If we consider graphs, all points are non characteristics. At these points several equivalent definitions of the horizontal mean curvature $h_{0}$ have been proposed. To quote a few: $h_{0}$ can be defined in terms of the first variation of the area functional $[16,15,19,26,37,13]$, as horizontal divergence of the horizontal unit normal or as limit of the mean curvatures $h_{\epsilon}$ of suitable Riemannian approximating metrics $\sigma_{\epsilon}$ [13]. If the surface is not regular, the notion of curvature can be expressed in the viscosity sense (we refer to [3], [4], [42], [41], [29], [2], [31], [6] for viscosity solutions of PDE in the sub-Riemannian setting).

The mean curvature flow of a graph in $G \times \mathbb{R}$ is characterized by the fact that each point of the evolving graph moves in the direction of the upward unit normal with speed equal to the mean curvature. In the setting of the approximating Riemannian metrics $\left(G, \sigma_{\epsilon}\right)$ this flow is smooth and in terms of the functions $t \rightarrow u_{\epsilon}(\cdot, t): \Omega \subset G \rightarrow \mathbb{R}$ describing the evolving graphs, the relevant equation reads:

$$
\frac{\partial u_{\epsilon}}{\partial t}=W_{\epsilon} h_{\epsilon}=W_{\epsilon} \sum_{i=1}^{n} X_{i}^{\epsilon}\left(\frac{X_{i}^{\epsilon} u_{\epsilon}}{W_{\epsilon}}\right)=\sum_{i, j=1}^{n} a_{i j}^{\epsilon}\left(\nabla_{\epsilon} u_{\epsilon}\right) X_{i}^{\epsilon} X_{j}^{\epsilon} u_{\epsilon} \quad \text { for } x \in \Omega, t>0,
$$

where, $h_{\epsilon}$ is the mean curvature of the graph of $u_{\epsilon}(\cdot, t)$ and

$$
W_{\epsilon}^{2}=1+\left|\nabla_{\epsilon} u_{\epsilon}\right|^{2}=1+\sum_{i=1}^{n}\left(X_{i}^{\epsilon} u_{\epsilon}\right)^{2} \text { and } a_{i j}^{\epsilon}(p)=\delta_{i j}-\frac{p_{i} p_{j}}{1+|p|^{2}},
$$

for all $p \in \mathbb{R}^{n}$. In the sub-Riemannian limit $\epsilon=0$ the equation reads

$$
\frac{\partial u}{\partial t}=\sqrt{1+\left|\nabla_{0} u\right|^{2}} \sum_{i=1}^{m} X_{i}\left(\frac{X_{i} u}{\sqrt{1+\left|\nabla_{0} u\right|^{2}}}\right)
$$

with $u:=\lim _{\epsilon \rightarrow 0} u_{\epsilon}$, for $x \in \Omega$ and $t>0$. Mean curvature flow in the setting of Carnot group has been studied by [6] and [12]. See also the recent [20] as well as [21] for a probabilistic interpretation of the flow.

The aim of this paper is to establish uniform (in the parameter $\epsilon$ as $\epsilon \rightarrow 0$ ) estimates and determine the asymptotic behavior of solutions to the initial value problem for the mean curvature motion of graphs over bounded domains of a group $G$,

$$
\begin{cases}\partial_{t} u_{\epsilon}=h_{\epsilon} W_{\epsilon} & \text { in } Q=\Omega \times(0, T) \\ u_{\epsilon}=\varphi & \text { on } \partial_{p} Q\end{cases}
$$

Here $\partial_{p} Q=(\Omega \times\{t=0\}) \cup(\partial \Omega \times(0, T))$ denotes the parabolic boundary of $Q$.

The classical parabolic theory yields local existence and uniqueness for smooth solutions $u_{\epsilon}$ of (1.6) of the problem, under suitable assumptions on the boundary data. Our main goal 
consists in proving that estimates are stable as $\epsilon$ tends to 0 , thus providing estimates also for the solution of the limit problem.

Our first result consists in showing that if the initial/boundary data is sufficiently smooth then the solutions of (1.6) are Lipschitz up to the boundary uniformly in $\epsilon>0$.

Theorem 1.2. (Global gradient bounds) Let $G$ be a Lie group of step two, $\Omega \subset G$ a bounded, open, convex set(in the sense of definition 3.3 below) and $\varphi \in C^{2}(\bar{\Omega})$. For $1 \geq \epsilon>0$ denote by $u_{\epsilon} \in C^{2}(\Omega \times(0, T)) \cap L^{\infty}\left((0, \infty), C^{1}(\bar{\Omega})\right)$ the non-negative unique solution of the initial value problem (1.6). There exists $C=C\left(G,\|\varphi\|_{C^{2}(\bar{\Omega})}\right)>0$ such that

$$
\sup _{\bar{\Omega} \times(0, T)}\left|\nabla_{1} u_{\epsilon}\right| \leq C \text {. }
$$

In particular, since $\sup _{\bar{\Omega} \times(0, T)}\left|\nabla_{\epsilon} u_{\epsilon}\right| \leq \sup _{\bar{\Omega} \times(0, T)}\left|\nabla_{1} u_{\epsilon}\right|$ one has uniform Lipschitz bounds for $u_{\epsilon}$.

Having established Lipschitz bounds, the next step is to recognize that right derivatives $X_{i}^{r} u_{\epsilon}$ of the solutions of (1.6) are solutions of a divergence form, degenerate parabolic PDE. We prove that weak solutions of such PDE satisfy a Harnack inequality and consequently obtain $C^{1, \alpha}$ interior estimates for the original solution $u_{\epsilon}$, which are uniform in $\epsilon>0$.

At this point one rewrites the PDE in (1.6) in non-divergence form and invokes the stable Schauder estimates for subriemannian equations (see [10], [7]) to prove local higher regularity and long time existence.

Theorem 1.3. In the hypothesis of Theorem 1.2 there exists a unique solution $u_{\epsilon} \in C^{\infty}(\Omega \times$ $(0, \infty)) \cap L^{\infty}\left((0, \infty), C^{1}(\bar{\Omega})\right)$ of the initial value problem

$$
\begin{cases}\partial_{t} u_{\epsilon}=W_{\epsilon} h_{\epsilon} & \text { in } Q=\Omega \times(0, \infty) \\ u_{\epsilon}=\varphi & \text { on } \partial_{p} Q\end{cases}
$$

and that for each $K \subset \subset \Omega$ and for each $h \in \mathbb{N}$ there exists $C_{h}=C_{h}(G, \varphi, h, K, \Omega)>0$ not depending on $\epsilon$ such that

$$
\left\|u_{\epsilon}\right\|_{C^{h}(K \times[0,+\infty[)} \leq C_{h}
$$

Since the estimates are uniform in $\epsilon$ and in time, and with respect to $\epsilon$, we will deduce the following corollary:

Corollary 1.4. Under the assumptions of the Theorem 1.2, as $\epsilon \rightarrow 0$ the solutions $u_{\epsilon}$ converge uniformly (with all its derivatives) on compact subsets of $Q$ to the unique, smooth solution $u_{0} \in C^{\infty}(\Omega \times(0, \infty)) \cap L^{\infty}\left((0, \infty), C^{1}(\bar{\Omega})\right)$ of the sub-Riemannian mean curvature flow $(1.5)$ in $\Omega \times(0, \infty)$ with initial data $\varphi$.

Corollary 1.5. Under the assumptions of Theorem 1.2, as $T \rightarrow \infty$ the solutions $u_{\epsilon}(\cdot, t)$ converge uniformly on compact subsets of $\Omega$ to the unique solution of the minimal surface equation

$$
h_{\epsilon}=0 \quad \text { in } \Omega
$$


with boundary value $\varphi$, while $u_{0}=\lim _{\epsilon \rightarrow 0} u_{\epsilon} \in C^{\infty}(\Omega) \cap \operatorname{Lip}(\bar{\Omega})$ is the unique solution of the sub-Riemannian minimal surfaces equation $h_{0}=0$ in $\Omega$, with boundary data $\varphi$.

Regularity of minimal surfaces in the special case of Heisenberg group has been investigated in $[23,36,16,15,8,9,18,33,37,39]$.

Acknowledgements We are grateful to the anonymous referee for the valuable feedback.

\section{Structure stability in the Riemannian Limit}

If $x \in G$ and $r>0$, we will denote by

$$
B(x, r)=\left\{y \in G \mid d_{0}(x, y)<r\right\}
$$

the balls in the Carnot-Carathéodory control distance corresponding to the subRiemannian metric $\sigma_{0}$. For each $\epsilon>0$ we also define the distance function $d_{\epsilon}$ corresponding to the Riemannian metric $\sigma_{\epsilon}$,

$$
\begin{aligned}
d_{\epsilon}(x, y)=\inf \left\{\int_{0}^{1}\left|\gamma^{\prime}\right|_{\sigma_{\epsilon}}(s) d s \text { with } \gamma:[0,1] \rightarrow G\right. \\
\text { a Lipschitz curve s. t. } \gamma(0)=x, \gamma(1)=y\} .
\end{aligned}
$$

Set

$$
B_{\epsilon}(x, r)=\left\{y \in G \mid d_{\epsilon}(x, y)<r\right\} .
$$

Note that in the definition of $d_{\epsilon}$, if the curve for which the infimum is achieved happens to be horizontal then $d_{\epsilon}(x, y)=d_{0}(x, y)$. In general we have $\sup _{\epsilon>0} d_{\epsilon}(x, y)=d_{0}(x, y)$ and it is well known that $\left(G, d_{\epsilon}\right)$ converges in the Gromov-Hausdorff sense as $\epsilon \rightarrow 0$ to the sub-Riemannian space $\left(G, d_{0}\right)$. (See for instance $[25]$ and references therein).

2.1. Stability of the homogenous structure as $\epsilon \rightarrow 0$. If we denote by $d x$ the Haar measure in $G$, and by $|\Omega|$ the corresponding measure of a subset $\Omega \subset G$, then Rea and two of the authors have proved in $[7,11]$ that

Proposition 2.1. There exist constants $C, R>0$ independent of $\epsilon$ such that for every $x \in G$ and $R>r>0$,

$$
\left|B_{\epsilon}(x, 2 r)\right| \leq C\left|B_{\epsilon}(x, r)\right|
$$

Having this property the spaces $\left(G, d_{\epsilon}, d x\right)$ are called homogenous with constant $C>0$ independent of $\epsilon$ (see [17]).

Let $\tau>0$ and consider the space $\tilde{G}=G \times(0, \tau)$ with its product Lebesgue measure $d x d t$. In $\tilde{G}$ define the pseudo-distance function

$$
\tilde{d}_{\epsilon}((x, t),(y, s))=\max \left(d_{\epsilon}(x, y), \sqrt{|t-s|}\right) .
$$

Proposition 2.1 tells us that $\left(\tilde{G}, \tilde{d}_{\epsilon}, d x d t\right)$ is a homogeneous space with constant independent of $\epsilon \geq 0$. Likewise, the Poincaré inequality holds for all $\epsilon$ near zero, with constant independent of $\epsilon$. 
2.2. Stability of Schauder estimates. Let us recall uniform estimates in spaces of Hölder continuous functions for solutions of second order sub-elliptic differential equations in non divergence form

$$
L_{\epsilon, A} u \equiv \partial_{t} u-\sum_{i, j=1}^{n} a_{i j}^{\epsilon}(x, t) X_{i}^{\epsilon} X_{j}^{\epsilon} u=0,
$$

in a cylinder $Q=\Omega \times(0, T)$ that are stable as $\epsilon \rightarrow 0$. We will assume ellipticity

$$
\Lambda^{-1}|\eta|^{2} \leq a_{i j}^{\epsilon}(x, t) \eta^{i} \eta^{j} \leq \Lambda|\eta|^{2},
$$

with $\Lambda>0$, and for a.e. $(x, t) \in Q$, all $\eta \in \mathbb{R}^{n}$ and $\epsilon \in[0,1]$.

Let us start with the definition of classes of Hölder continuous functions in this setting

Definition 2.2. Let $0<\alpha<1, Q \subset \mathbb{R}^{n+1}$ and $u$ be defined on $Q$. We say that $u \in C_{\epsilon, X}^{\alpha}(Q)$ if there exists a positive constant $M$ such that for every $(x, t),\left(x_{0}, t_{0}\right) \in Q$

$$
\left|u(x, t)-u\left(x_{0}, t_{0}\right)\right| \leq M \tilde{d}_{\epsilon}^{\alpha}\left((x, t),\left(x_{0}, t_{0}\right)\right)
$$

We put

$$
\|u\|_{C_{\epsilon, X}^{\alpha}(Q)}=\sup _{(x, t) \neq\left(x_{0}, t_{0}\right)} \frac{\left|u(x, t)-u\left(x_{0}, t_{0}\right)\right|}{\tilde{d}_{\epsilon}^{\alpha}\left((x, t),\left(x_{0}, t_{0}\right)\right)}+\sup _{Q}|u| .
$$

Iterating this definition, if $k \geq 1$ we say that $u \in C_{\epsilon, X}^{k, \alpha}(Q)$ if for all $i=1, \ldots, m X_{i} u \in$ $C_{\epsilon, X}^{k-1, \alpha}(Q)$. Where we have set $C_{\epsilon, X}^{0, \alpha}(Q)=C_{\epsilon, X}^{\alpha}(Q)$.

Internal Schauder estimates for these type of operators are well known. We recall the results of Capogna and Han [14] for uniformly subelliptic operators, of Bramanti and Brandolini [5] for heat-type operators, and the results of Lunardi [30] and Gutiérrez and Lanconelli [24], which apply to a large class of squares of vector fields plus a drift term. Schauder estimates uniform in $\epsilon$ have been proved by the authors in [10] in the setting of Carnot Groups and by two of us in [7] in the setting of general Hörmander type vector fields.

These result can be stated as

Proposition 2.3. Let $w$ be a smooth solution of $L_{\epsilon, A} w=f$ on $Q$. Let $K$ be a compact sets such that $K \subset \subset Q$, set $2 \delta=d_{0}\left(K, \partial_{p} Q\right)$ and denote by $K_{\delta}$ the $\delta$-tubular neighborhood of $K$. Assume that there exists a constant $C>0$ such that

$$
\left\|a_{i j}^{\epsilon}\right\|_{C_{\epsilon, X}^{k, \alpha}\left(K_{\delta}\right)} \leq C
$$

for any $\epsilon \in(0,1)$. There exists a constant $C_{1}>0$ depending on $\alpha, C, \delta$ but independent of $\epsilon$, such that

$$
\|w\|_{C_{\epsilon, X}^{k+2, \alpha}(K)} \leq C_{1}\left(\|f\|_{C_{\epsilon, X}^{k, \alpha}\left(K_{\delta}\right)}+\|w\|_{C_{\epsilon, X}^{k+1, \alpha}\left(K_{\delta}\right)}\right) .
$$




\section{Gradient estimates}

In this section we prove Theorem 1.2. The proof is carried out in two steps: First we use the maximum principle to establish interior $L^{\infty}$ bounds for the full gradient of the solution $\nabla_{1} u$ of (1.6) with respect to the Lipschitz norm of $u$ on the parabolic boundary. Next, we construct appropriate barriers and invoke the comparison principle established in [6] to prove boundary gradient estimates. The combination of the two will yield the uniform global Lipschitz bounds.

3.1. Interior gradient estimates. Recalling that the right invariant vector fields $X_{j}^{r}$ commute with the left invariant frame $X_{i}, i=1, \ldots, n$ it is easy to show through a direct computation the following result.

Lemma 3.1. Let $u_{\epsilon} \in C^{3}(Q)$ be a solution to (1.3) and denote $v_{0}=\partial_{t} u_{\epsilon}, v_{i}=X_{i}^{r} u_{\epsilon}$ for $i=i, \ldots, n$. Then for every $h=0, \ldots, n$ the function $v_{h}$ is a solution of

$$
-\partial_{t} v_{h}+\sum_{i, j, k=1}^{n} \frac{\partial a_{i j}^{\epsilon}}{\partial p_{k}}\left(\nabla_{\epsilon} u\right) X_{i}^{\epsilon} X_{j}^{\epsilon} u_{\epsilon} X_{k}^{\epsilon} v_{h}+\sum_{i, j=1}^{n} a_{i j}^{\epsilon}\left(\nabla_{\epsilon} u\right) X_{i}^{\epsilon} X_{j}^{\epsilon} v_{h}=0 .
$$

Note that in order to prove $L^{\infty}$ bounds on the horizontal gradient of solutions of (1.6) one cannot invoke Lemma 3.1 with differentiation along the horizontal left invariant frame, because such vector fields do not commute.

Let us explicitly note that the right derivatives $X_{k}^{1, r}$ are a basis of the tangent space, as well as $X_{k}^{1}$, so that it is possible to represent each family of vector fields as linear combination of the other. In particular, in the Carnot setting, it has been proved in [38] that there exist homogenous polynomials $c_{k j}$ such that

$$
X_{k}^{1}=\sum_{j} c_{k j} X_{j}^{1, r}
$$

In the general Lie groups setting this assertion is true only locally and the functions $c_{k j}$ are polynomials in the local exponential variables independent of $\epsilon$.

In view of this observation and from the weak maximum principle one may easily deduce that:

Proposition 3.2. Let $u_{\epsilon} \in C^{3}(Q)$ be a solution to (1.6) with $\Omega$ bounded. There exists $C=C\left(G,\|\varphi\|_{C^{2}(\Omega)}\right)>0$ such that

$$
\sup _{Q}\left|\nabla_{1} u_{\epsilon}\right| \leq \sup _{\partial_{p} Q}\left(\left|\nabla_{1} u_{\epsilon}\right|+\left|\partial_{t} u_{\epsilon}\right|\right)
$$

where $\nabla_{1}$ is the full $\sigma_{1}-$ Riemannian gradient.

3.2. Barrier functions and boundary gradient estimate. In [6, Section 4.2] it is shown that in a step two Carnot group coordinate planes (i.e. images under the exponential of level sets of the form $x_{k}=0$ ) solve the minimal surface equation $h_{0}=0$. In the same paper it is also shown that this may fail for step three or higher. In order to adapt the construction 
of the barrier to the present non-nilpotent setting we will need a refinement of this result, based on the following definition of convex set:

Definition 3.3. For every point $x_{0} \in \partial \Omega$ consider the canonical coordinates $\Phi_{x_{0}}(u)$ defined in (1.1) and centered at $x_{0}$ (so that $x_{0}$ is represented by the origin in these coordinates). Assume that $\Omega$ has a tangent plane $\Pi$ at the point $u=0$ and assume that $\Phi_{x_{0}}^{-1}(\Omega)$ is lying on one side of the plane. If this happens for every $x_{0} \in \partial \Omega$ we say that $\Omega$ is convex.

In a step two Carnot group this definition is equivalent to the one in [10], and every set that is Euclidean convex when expressed in exponential coordinates satisfies the condition. Using Darboux coordinates one can see that the same holds for the root-translation group $\mathcal{R} \mathcal{T}$. This ad-hoc definition of convexity is not implied, and does not imply, the notion of horizontal convexity as defined for instance in [29].

Lemma 3.4. Let $G$ be a step two Carnot group. If $f: G \rightarrow \mathbb{R}$ is linear (in exponential coordinates) then for every $\epsilon \geq 0$, the matrix with entries $X_{i}^{\epsilon} X_{j}^{\epsilon} f$ is anti-symmetric, in particular every level set of $f$ satisfies $h_{\epsilon}=0$.

The previous lemma and the Rothschild and Stein local osculation result will lead to the construction of a barrier in the present setting, and to establish a priori Lipschitz estimates at the boundary for solutions. We begin by recalling an immediate consequence of the proof of [6, Theorem 3.3].

Lemma 3.5. For each $\epsilon \geq 0$, if $v_{\epsilon}$ is a bounded subsolution and $w_{\epsilon}$ is a bounded supersolution of (1.6) then $v_{\epsilon}(x, t) \leq w_{\epsilon}(x, t)$ for all $(x, t) \in Q$.

Let $u_{\epsilon} \in C^{2}(Q)$ be a solution of (1.6), and express the evolution PDE in non-divergence form

$$
\partial_{t} u_{\epsilon}=h_{\epsilon} W_{\epsilon}=a_{i j}^{\epsilon}\left(\nabla_{\epsilon} u\right) X_{i}^{\epsilon} X_{j}^{\epsilon} u_{\epsilon}
$$

Set $v_{\epsilon}=u_{\epsilon}-\varphi$ so that $v_{\epsilon}$ solves the homogenous 'boundary' value problem

$$
\begin{cases}\partial_{t} v_{\epsilon}=a_{i j}^{\epsilon}\left(\nabla_{\epsilon} v_{\epsilon}+\nabla_{\epsilon} \varphi\right) X_{i}^{\epsilon} X_{j}^{\epsilon} v_{\epsilon}+b^{\epsilon} & \text { in } Q=\Omega \times(0, T) \\ v_{\epsilon}=0 & \text { on } \partial_{p} Q,\end{cases}
$$

with $b^{\epsilon}(x)=a_{i j}^{\epsilon}\left(\nabla_{\epsilon} v_{\epsilon}(x)+\nabla_{\epsilon} \varphi(x)\right) X_{i}^{\epsilon} X_{j}^{\epsilon} \varphi(x)$. We define our (weakly) parabolic operator for which the function $v_{\epsilon}$ is a solution

$$
P(v)=a_{i j}^{\epsilon}\left(\nabla_{\epsilon} v_{\epsilon}+\nabla_{\epsilon} \varphi\right) X_{i}^{\epsilon} X_{j}^{\epsilon} v_{\epsilon}+b^{\epsilon}-\partial_{t} v .
$$

In the following we construct for each point $p_{0}=\left(x_{0}, t_{0}\right) \in \partial \Omega \times(0, T)$ a barrier function for $Q, v_{\epsilon}$ : i.e.,

Lemma 3.6. Let $G$ be a Lie group free up to step two and $\Omega \subset G$ convex in the sense of Definition 3.3. For each point $p_{0}=\left(x_{0}, t_{0}\right) \in \partial \Omega \times(0, T)$ and for every $\epsilon>0$ there exist a parabolic neighborhood $V_{\epsilon}$ of $p_{0}$ and a positive function $w_{\epsilon} \in C^{2}(Q)$ such that

$$
P\left(w_{\epsilon}\right) \leq 0 \text { in } V_{\epsilon} \cap Q \text { with } w_{\epsilon} \geq v_{\epsilon} \text { in } \partial_{p} V_{\epsilon} \cap Q \text {. }
$$


Proof. For every $x_{0} \in \partial \Omega$ we can select exponential coordinates locally around the point $x_{0}$. The point $x_{0}$ has coordinates 0 in the variables $u$.

In these coordinates there exists an hyperplane tangent to the open set $\Omega$ defined by an equation of the form $\Pi(u)=\sum_{i=1}^{n} a_{i} u_{i}=0$ with $\Pi>0$ in $\Omega, \Pi(0)=0$, and normalized as $\sum_{d(i)=1,2} a_{i}^{2}=1$. Following the standard argument (see for instance [28, Chapter 10]) we select the barrier at $\left(x_{0}, t_{0}\right) \in \partial \Omega \times(0, T)$ independent of time with

$$
\tilde{w}_{\epsilon}=\psi(\Pi)
$$

with $\psi$ solution of

$$
\psi^{\prime \prime}+\nu\left(\psi^{\prime}\right)^{2}=0
$$

in particular

$$
\psi(s)=\frac{1}{\nu} \log (1+k s),
$$

with $k$ and $\nu$ chosen appropriately so that conditions (3.6) will hold. We choose a neighborhood $V=O \times(0, T)$ such that $\Pi \cap O \cap \partial \Omega=\left\{x_{0}\right\}$. By an appropriate choice of $k$ sufficiently large we can easily obtain $\tilde{w}_{\epsilon}(0)=0$ and $\tilde{w}_{\epsilon} \circ \Theta_{x_{0}} \geq v_{\epsilon}$ in $\partial_{p} V \cap Q$.

We denote by $P_{Y}$ the operator which has the same expression of $P$, but with respect to the left invariant osculating frame $\left\{Y_{i}^{\epsilon}\right\}_{i=1, \ldots, n}$ in the nilpotent osculating free group $G_{m, 2}$, i.e. $P_{Y}(v)=a_{i j}^{\epsilon}\left(\nabla_{Y, \epsilon} v_{\epsilon}+\nabla_{Y, \epsilon} \varphi\right) Y_{i}^{\epsilon} Y_{j}^{\epsilon} v_{\epsilon}+b^{\epsilon}-\partial_{t} v$.

To estimate $P_{Y}\left(\tilde{w}_{\epsilon}\right) \leq 0$ we begin by observing that $\tilde{w}_{\epsilon}$ satisfies

$$
P_{Y}\left(\tilde{w}_{\epsilon}\right)=\psi^{\prime} a_{i j}^{\epsilon}\left(\nabla_{Y, \epsilon} \tilde{w}+\nabla_{Y, \epsilon} \varphi\right) Y_{i}^{\epsilon} Y_{j}^{\epsilon} \Pi+\frac{\psi^{\prime \prime}}{\left(\psi^{\prime}\right)^{2}} \mathcal{F}+b_{\epsilon},
$$

with $\mathcal{F}=a_{i j}^{\epsilon}\left(\nabla_{Y, \epsilon} \tilde{w}_{\epsilon}+\nabla_{Y, \epsilon}\left(\varphi \circ \Phi_{x_{0}}\right) Y_{i}^{\epsilon} \tilde{w}_{\epsilon} Y_{j}^{\epsilon} \tilde{w}_{\epsilon}\right.$.

We first note that

$$
a_{i j}^{\epsilon}\left(\nabla_{Y, e} \tilde{w}_{\epsilon}+\nabla_{Y, \epsilon}\left(\varphi \circ \Phi_{x_{0}}\right)\right)\left(Y_{i}^{\epsilon} Y_{j}^{\epsilon}\right) \Pi=0
$$

as $a_{i j}^{\epsilon}$ is symmetric and $X_{i}^{\epsilon} X_{j}^{\epsilon} \Pi$ is anti-symmetric in view of Lemma 3.4. We can now estimate the remaining terms of (3.10)

$$
\frac{\psi^{\prime \prime}}{\left(\psi^{\prime}\right)^{2}} \mathcal{F}+b_{\epsilon}
$$

in a parabolic neighborhood of $u=0$. We first note that Lemma 3.4 implies

$$
\begin{gathered}
\frac{\epsilon^{2}}{2} \leq \max \left(\sum_{d(i)=1} a_{i}^{2}, \epsilon^{2} \sum_{d(k)=1} a_{k}^{2}\right) \leq\left|\nabla_{Y, \epsilon} \Pi\right|= \\
=\sum_{d(i)=1}\left(a_{i}+\sum_{d(k)=2, d(j)=1} c_{i j}^{k} a_{k} x_{j}\right)^{2}+\epsilon^{2} \sum_{d(k)=2} a_{k}^{2} \leq C(G)\left(1+\epsilon^{2}\right),
\end{gathered}
$$


for some constant $C(G)>0$. Consequently, for $\psi^{\prime}>>1$ sufficiently large one finds

$$
\mathcal{F} \geq \frac{\left|\nabla_{Y, \epsilon} \tilde{w}_{\epsilon}\right|^{2}}{1+\left|\nabla_{Y, \epsilon} \tilde{w}_{\epsilon}+\nabla_{Y, \epsilon} \varphi\right|^{2}} \geq C(G) \frac{\left|\nabla_{Y, \epsilon} \tilde{w}_{\epsilon}\right|^{2}}{1+\left|\psi^{\prime}\right|^{2}+\left|\nabla_{Y, \epsilon} \varphi\right|^{2}} \geq C(G) \epsilon^{2}>0
$$

with $C(G)>0$ a constant depending only on $G$ (not always the same along the chain of inequalities). In view of the definition of $b_{\epsilon}$ and (3.8) with an appropriate choice of $\nu=\nu(G, \epsilon, \phi)>0$ and $k=k(G, \phi)>>1$ in (3.9), we conclude

$$
\frac{\Phi^{\prime \prime}}{\left(\Phi^{\prime}\right)^{2}} \mathcal{F}+b_{\epsilon} \leq\left(\frac{\psi^{\prime \prime}}{\left(\psi^{\prime}\right)^{2}}+\nu-1\right) \mathcal{F} \leq-C(G) \epsilon^{2}
$$

It follows that

$$
P_{Y}\left(\tilde{w}_{\epsilon}\right) \leq-C(G) \epsilon^{2}
$$

To conclude, we set $w_{\epsilon}=\tilde{w}_{\epsilon} \circ \Theta_{x_{0}}$. In view of the relation (1.2) between the vector fields $X$ and $Y$, it is now immediate to see that there exists a neighborhood $V_{\epsilon}$ of $p_{0}$, depending on $\epsilon$, such that

$$
P\left(w_{\epsilon}\right) \leq 0 \text { in } V_{\epsilon} \cap Q
$$

Proposition 3.7. Let $G$ be a Lie group free up to step two, $\Omega \subset G$ convex in the sense of Definition 3.3 and $\varphi \in C^{2}(\bar{\Omega})$. For $\epsilon>0$ denote by $u_{\epsilon} \in C^{2}(\Omega \times(0, T)) \cap C^{1}(\bar{\Omega} \times$ $(0, T))$ the non-negative unique solution of the initial value problem (1.6). There exists $C=C\left(G,\|\varphi\|_{C^{2}(\bar{\Omega})}\right)>0$ such that

$$
\sup _{\partial \Omega \times(0, T)}\left|\nabla_{\epsilon} u_{\epsilon}\right| \leq \sup _{\partial \Omega \times(0, T)}\left|\nabla_{1} u_{\epsilon}\right| \leq C .
$$

Proof. In view of Lemma 3.5, a comparison with the barrier constructed above yields that

$$
0 \leq \frac{v_{\epsilon}(x, t)}{\operatorname{dist}_{\sigma_{1}}\left(x, x_{0}\right)} \leq \frac{w_{\epsilon}(x, t)}{\operatorname{dist}_{\sigma_{1}}\left(x, x_{0}\right)} \leq C(k, \nu),
$$

in $V_{\epsilon} \cap Q$, with dist $_{\sigma_{1}}\left(x, x_{0}\right)$ being the distance between $x$ and $x_{0}$ in the Riemannian metric $\sigma_{1}$, concluding the proof of the boundary gradient estimates at the point $p_{0}$.

\section{Regularity PRoperties in the $C^{k, \alpha}$ SPACES}

In this section we will prove uniform estimates for solution of (1.3) in the $C_{\epsilon, X}^{k, \alpha}$ Hölder spaces. This is accomplished in two steps and follows a strategy originally introduced by Trudinger (see notes in [28, Chapter 7]). First we establish $C^{1, \alpha}$ regularity, 


\subsection{Regularity properties in the $C^{1, \alpha}$ spaces.}

Remark 4.1. Let $u$ be a smooth solution of (1.3). Note that the function $v_{k}=X_{k}^{r} u$ is then a solution of the equation

$$
-\partial_{t} v_{k}+\sum_{i, j=1}^{n} X_{i}^{\epsilon}\left(a_{i j}^{\epsilon}\left(\nabla_{\epsilon} u\right) X_{j}^{\epsilon} v_{k}\right)+\sum_{i, j, h=1}^{n} a^{i, j, h} X_{i}^{\epsilon} X_{j}^{\epsilon} u_{\epsilon} X_{h}^{\epsilon} v_{k}=0,
$$

where

$$
a^{i, j, h}=\frac{\partial a_{i j}^{\epsilon}}{\partial p_{h}}-\frac{\partial a_{i h}^{\epsilon}}{\partial p_{j}} .
$$

Indeed the equation provided in (3.1) in non divergence form can be re-written in divergence form as

$$
-\partial_{t} v_{k}+\sum_{i, j=1}^{n} X_{i}^{\epsilon}\left(a_{i j}^{\epsilon}\left(\nabla_{\epsilon} u\right) X_{j}^{\epsilon} v_{k}\right)+\sum_{i, j, h=1}^{n} \frac{\partial a_{i j}^{\epsilon}}{\partial p_{h}} X_{i}^{\epsilon} X_{j}^{\epsilon} u_{\epsilon} X_{h}^{\epsilon} v_{k}-\sum_{i, j, h=1}^{n} \frac{\partial a_{i j}^{\epsilon}}{\partial p_{h}} X_{j}^{\epsilon} X_{k}^{r} u_{\epsilon} X_{i}^{\epsilon} X_{h}^{\epsilon} u_{\epsilon}=0
$$

and the latter is equivalent to (4.1).

Remark 4.2. Starting from (4.1) one can immediately see that the function $z=\left|\nabla_{\epsilon}^{r} u_{\epsilon}\right|^{2}$ is solution of

$$
-\partial_{t} z+\sum_{i, j=1}^{n} X_{i}^{\epsilon}\left(a_{i j}^{\epsilon}\left(\nabla_{\epsilon} u\right) X_{j}^{\epsilon} z\right)+\sum_{i, j, h=1}^{n} a^{i j h} X_{i}^{\epsilon} X_{j}^{\epsilon} u_{\epsilon} X_{h}^{\epsilon} z-2 \sum_{i, j, h, k=1}^{n} a_{i j}^{\epsilon} X_{i}^{\epsilon} v_{k} X_{j}^{\epsilon} v_{k}=0 .
$$

Lemma 4.3. for every $k=1, \ldots, n$ and for every $\delta>0$ the functions

$$
w_{k}^{ \pm}= \pm v_{k}+\delta z
$$

satisfy the inequality

$$
-\partial_{t} w_{k}^{ \pm}+\sum_{i, j=1}^{n} X_{i}^{\epsilon}\left(a_{i j}^{\epsilon}\left(\nabla_{\epsilon} u\right) X_{j}^{\epsilon} w_{k}^{ \pm}\right) \geq-C_{0}\left|\nabla_{\epsilon} w_{k}^{ \pm}\right|^{2}-C_{1},
$$

for suitable constants $C_{0}$ and $C_{1}$.

Proof. For simplicity we temporarily drop the \pm superscript. Adding the equations satisfied by $v_{k}$ and $z$ we see that for every $k=1, \ldots, n$ and for every $\delta>0$ the functions $w_{k}^{ \pm}= \pm v_{k}+\delta z$ satisfy

$$
\begin{gathered}
-\partial_{t} w_{k}+\sum_{i, j=1}^{n} X_{i}^{\epsilon}\left(a_{i j}^{\epsilon}\left(\nabla_{\epsilon} u\right) X_{j}^{\epsilon} w_{k}\right)=-\sum_{i, j, h=1}^{n} a^{i j h} X_{i}^{\epsilon} X_{j}^{\epsilon} u_{\epsilon} X_{h}^{\epsilon} w_{k}+2 \delta \sum_{i, j, s=1}^{n} a_{i j}^{\epsilon} X_{i}^{\epsilon} v_{s} X_{j}^{\epsilon} v_{s} \\
\geq 2 \delta \lambda \sum_{s=1}^{n}\left|\nabla_{\epsilon} X_{s}^{1, r} u_{\epsilon}\right|^{2}-\sup _{i, j, h}\left|a^{i j h}\right|\left|X_{i}^{\epsilon} X_{j}^{\epsilon} u_{\epsilon}\right|\left|\nabla_{\epsilon} w_{k}\right|
\end{gathered}
$$


where $\lambda>0$ is the smallest eigenvalue of $a_{i j}^{\epsilon}$ and is independent of $\epsilon>0$. Using the notation introduced in (3.2) we deduce that

$$
\left|X_{i}^{\epsilon} X_{j}^{\epsilon} u_{\epsilon}\right|=\left|\sum_{j, s=1}^{n} X_{i}^{\epsilon}\left(c_{s j} X_{s}^{1, r} u_{\epsilon}\right)\right| \leq C_{2}+C_{3} \sum_{s=1}^{n}\left|\nabla_{\epsilon} X_{s}^{1, r} u_{\epsilon}\right|
$$

Consequently, using Schwarz's inequality,

$$
\begin{gathered}
-\partial_{t} w_{k}+\sum_{i j} X_{i}\left(a_{i j}^{\epsilon}\left(\nabla_{\epsilon} u\right) X_{j}^{\epsilon} w_{k}\right) \geq \\
\geq 2 \delta \lambda \sum_{s=1}^{n}\left|\nabla_{\epsilon} X_{s}^{1, r} u_{\epsilon}\right|^{2}-\sum_{s=1}^{n} \delta \lambda\left|\nabla_{\epsilon} X_{s}^{1} u_{\epsilon}\right|^{2}-C_{0}\left|\nabla_{\epsilon} w_{k}\right|^{2}-C_{1},
\end{gathered}
$$

completing the proof.

Next we set $\left(x_{0}, t_{0}\right) \in Q=\Omega \times(0, T)$ and for $r>0$, let $Q_{\epsilon}(r)=\left\{(x, t) \in Q \mid d_{\epsilon}\left(x, x_{0}\right)<r\right.$ and $\left.\left|t-t_{0}\right| \leq r^{2}\right\}$. Define

and observe that

$$
W_{k}^{ \pm}=\sup _{Q_{\epsilon}(4 r)} w_{k}^{ \pm}
$$

$$
\partial_{t}\left(W_{k}^{ \pm}-w_{k}^{ \pm}\right)-\sum_{i, j=1}^{n} X_{i}^{\epsilon}\left(a_{i j}^{\epsilon}\left(\nabla_{\epsilon} u\right) X_{j}^{\epsilon}\left(W_{k}^{ \pm}-w_{k}^{ \pm}\right)\right) \geq-C_{0}\left|\nabla_{\epsilon}\left(W_{k}^{ \pm}-w_{k}^{ \pm}\right)\right|^{2}-C_{1} .
$$

In order to invoke the weak Harnack inequality and derive the $C^{\alpha}$ estimates, we need to eliminate the quadratic term on the right hand side. Following [28, Chapter 12, Sec. 3] we define

$$
\bar{w}_{k}=\frac{\lambda}{2 C_{0}}\left(1-\exp \left(\frac{2 C_{0}}{\lambda}\left(w_{k}^{ \pm}-W_{k}^{ \pm}\right)\right)\right)
$$

and observe that this new functions satisfies

$$
-\partial_{t} \bar{w}_{k}+\sum_{i j} X_{i}^{\epsilon}\left(a_{i j}^{\epsilon}\left(\nabla_{\epsilon} u\right) X_{j}^{\epsilon} \bar{w}_{k}\right)+g \leq 0
$$

where $g=C_{1}\left(\frac{2 C_{0}}{\lambda} \bar{w}_{k}+1\right)$, for the constants $\lambda, C_{0}, C_{1}$ from Lemma 4.3. In view of the weak Harnack inequality [7, Proposition 7.6], one has that for some constant $C_{4}>0$ independent of $\epsilon$ and for $Q_{\epsilon}^{-}(r)=\left\{(x, t) \in Q \mid d_{\epsilon}\left(x, x_{0}\right)<r\right.$ and $\left.t_{0}-3 r^{2}<t<t_{0}<2 t^{2}\right\}$,

$$
\int_{Q_{\epsilon}^{-}(r)} \bar{w}_{k} d x d t \leq C_{4}\left(\inf _{Q_{\epsilon}(r)} \bar{w}_{k}+\sup _{Q_{\epsilon}(r)}|g| r^{2}\right) .
$$

Following the argument in [28, Chapter 12, Sec. 3] we obtain

Proposition 4.4. Let $u_{\epsilon}$ be a solution of the mean curvature flow PDE (1.3) in $Q=\Omega \times$ $(0, T) \subset G \times \mathbb{R}$. Let $K$ be a compact sets such that $K \subset \subset Q$, set $2 \delta=d_{0}\left(K, \partial_{p} Q\right)$ and denote by $K_{\delta}$ the $\delta$-tubular neighborhood of $K$ in $d_{0}$. There exists constants $C>0$ and $\alpha \in(0,1)$ depending on $\delta$ and on the Lipschitz norm of $u$ in $K_{\delta}$, but independent of $\epsilon$, such that

$$
\left\|u_{\epsilon}\right\|_{C_{\epsilon, X}^{1, \alpha}\left(K_{\delta}\right)} \leq C
$$


4.2. Regularity properties in the $C^{k, \alpha}$ spaces. Once obtained the interior $C^{1, \alpha}$ estimate of the solution uniform in $\epsilon$, we write the mean curvature flow equation in non divergence form:

$$
\partial_{t} u-\sum_{i, j=1}^{n} a_{i j}^{\epsilon}(x, t) X_{i}^{\epsilon} X_{j}^{\epsilon} u=0 .
$$

Applying Schauder estimates in Proposition 2.3 we immediately deduce the proof of Theorem 1.3 .

Proof of Theorem 1.3. Since the solution is of class $C^{1, \alpha}$, and the norm is bounded uniformly in $\epsilon$ then $u_{\epsilon}$ it is a solution of a divergence form equation

$$
\partial_{t} u_{\epsilon}-\sum_{i, j=1}^{n} a_{i j}^{\epsilon}(x, t) X_{i}^{\epsilon} X_{j}^{\epsilon} u_{\epsilon}=0,
$$

with $a_{i j}^{\epsilon}$ of class $C^{\alpha}$ such that for every $K$ be a compact sets such that $K \subset \subset Q$ and $2 \delta=d_{0}\left(K, \partial_{p} Q\right)$ there exists a positive constant $C_{0}$ such that

$$
\left\|a_{i j}^{\epsilon}\right\|_{C_{\epsilon, X}^{\alpha}\left(K_{\delta}\right)} \leq C_{0}
$$

for every $\epsilon \in(0,1)$. Consequently, by Proposition 2.3 there exists a constant $C_{2}$ such that

$$
\left\|u_{\epsilon}\right\|_{C^{2}(K)} \leq C_{2} \text {. }
$$

The conclusion immediately follows by induction.

\section{REFERENCES}

[1] D. G. Aronson, J. Serrin, Local behavior of solutions of quasilinear parabolic equations. Arch. Rational Mech. Anal., 25 (1967), 81-122.

[2] Z. M. Balogh, M. Rickly, Regularity of convex functions on Heisenberg groups. Ann. Sc. Norm. Super. Pisa Cl. Sci. (5) 2, 4 (2003), 847-868.

[3] T. Bieske, On 1-harmonic functions on the Heisenberg group. Comm. Partial Differential Equations 3-4, 27 (2002), 727-761.

[4] T. Bieske, Comparison principle for parabolic equations in the Heisenberg group, Electron. J. Differential Equations (2005), No. 95, 11 pp. (electronic).

[5] M. Bramanti, L. Brandolini, Schauder estimates for parabolic nondivergence operators of Hörmander type. J. Differential Equations 234, 1 (2007), 177-245.

[6] L. Capogna and G. Citti, Generalized mean curvature flow in Carnot groups, Comm. Partial Differential Equations 34 (2009), no. 7-9, 937-956.

[7] L. Capogna, G. Citti, Regularity for subelliptic P.D.E. through uniform estimates in multi-scale geometries, preprint (2015) available at arXiv:1504.04762.

[8] L. Capogna, G. Citti, M. Manfredini, Regularity of non-characteristic minimal graphs in the Heisenberg group $H^{1}$, Indiana Univ. Math. J. 58 (5) (2009) 2115-2160.

[9] L. Capogna, G. Citti, M. Manfredini, Smoothness of Lipschitz intrinsic minimal graphs in the Heisenberg group $H^{n}, n>1 \mathrm{~J}$. Reine Angew. Math. (Crelles Journal) (2010), 648, 75-110.

[10] L. Capogna, G. Citti, M. Manfredini, Uniform Gaussian bounds for subelliptic heat kernels and an application to the total variation flow of graphs over Carnot groups, Analysis and Geometry in Metric Spaces 1, 2013 255-275. 
[11] L. Capogna, G. Citti, G. Rea, A subelliptic analogue of Aronson-Serrin's Harnack inequality, Math. Ann. 357 (2013), no. 3, 1175-1198.

[12] L. Capogna, G. Citti, C. Senni Guidotti Magnani, Sub-Riemannian heat kernels and mean curvature flow of graphs, J. Funct. Anal. 264 (2013), no. 8, 1899-1928.

[13] L. Capogna, D. Danielli, S. Pauls, J. Tyson, An introduction to the Heisenberg group and the subRiemannian isoperimetric problem, Progress in Mathematics, vol. 259, Birkhauser Verlag, Basel, 2007.

[14] L. Capogna, Q. Han, Pointwise Schauder estimates for second order linear equations in Carnot groups. In Harmonic analysis at Mount Holyoke (South Hadley, MA, 2001), vol. 320 of Contemp. Math. Amer. Math. Soc., Providence, RI, (2003), 45-69.

[15] J.-H. Cheng, J.-F. Hwang and P. Yang, Regularity of $C^{1}$ smooth surfaces with prescribed p-mean curvature in the Heisenberg group, Math. Ann. 344 (2009), no. 1, 1-35.

[16] J.-H. Cheng, J.-F. Hwang, A. Malchiodi and P. Yang, Minimal surfaces in pseudohermitian geometry, Ann. Sc. Norm. Super. Pisa Cl. Sci. (5) 4 (2005), 129-177.

[17] R. Coifman, G. Weiss, Analyse harmonique non-commutative sur certains espaces homognes. (french) Etude de certaines intégrales singulières, Lecture Notes in Mathematics, Vol. 242, Springer-Verlag, 1971.

[18] D. Danielli, N. Garofalo, D.M. Nhieu, S.D: Pauls: Instability of graphical strips and a positive answer to the Bernstein problem in the Heisenberg group $H^{1}$. J. Differ. Geom, (2009), 81(2), 251-295.

[19] D. Danielli, N. Garofalo, D.M. Nhieu, Sub-Riemannian calculus on hypersurfaces in Carnot groups, Adv. Math. 215 (2007), no. 1, 292-378

[20] N. Dirr, S. Dragoni, M. von Renesse, Evolution by mean curvature flow in sub-Riemannian geometries. Communications on Pure and Applied Mathematics, 9 (2), (2010) pp. 307-326.

[21] F. Ferrari, J. Manfredi, Q. Liu, On the horizontal Mean Curvature Flow for Axisymmetric surfaces in the Heisenberg Group, Commun. Contemp. Math. 16 (2014), no. 3.

[22] G. B. Folland, Subelliptic estimates and function spaces on nilpotent Lie groups, Ark. Mat. 2 (1975), no. $13,161-207$.

[23] N. Garofalo, D. M. Nhieu, Isoperimetric and Sobolev inequalities for Carnot-Carathéodory spaces and the existence of minimal surfaces, Comm. Pure Appl. Math., 49 (1996), no. 10, 1081-1144.

[24] C. Gutiérrez, E. Lanconelli, Maximum principle, nonhomogeneous Harnack inequality, and Liouville theorems for X-elliptic operators. Comm. Partial Differential Equations 28, 11-12 (2003), 1833-1862.

[25] M. Gromov, Metric structures for Riemannian and non-Riemannian spaces, Birkhauser Boston Inc., Boston, MA, 1999, Edited by J. LaFontaine and P. Pansu. Based on the 1981. French original, Translated from the French by Sean Michael Bates.

[26] Hladky, R. K. and Pauls, S. D. ,Constant mean curvature surfaces in sub-Riemannian geometry, J. Diff. Geom. 79 (2008) no.1, 111-139.

[27] L. Hörmander, Hypoelliptic second order differential equations, Acta Math. (1967), no. 119, 147-171.

[28] G. M. Lieberman, Second order parabolic differential equations, World Scientific Publishing Co. Inc., River Edge, NJ, 1996.

[29] G. Lu, J. J. Manfredi, B. Stroffolini, Convex functions on the Heisenberg group. Calc. Var. Partial Differential Equations 19, 1 (2004), 1-22.

[30] A. Lunardi, Analytic semigroups and optimal regularity in parabolic problems. Modern Birkhauser Classics. Birkhauser/Springer Basel AG, Basel, 1995.

[31] V. Magnani, Lipschitz continuity, Aleksandrov theorem, and characterizations for H-convex functions. Math. Ann. 334 (2006), 199-233.

[32] R. Montgomery, A tour of sub-Riemannian geometries, their geodesics and applications. No. 91 in Mathematical Surveys and Monographs. American Mathematical Society, 2002

[33] R. Monti, M. Rickly, Convex isoperimetric sets in the Heisenberg group. Ann. Sc. Norm. Super. Pisa Cl. Sci. (5) 8 (2009), no. 2, 391-415. 
[34] J. Moser, A Harnack inequality for parabolic differential equations, Comm. Pure Appl. Math. 17 (1964), 101-134.

[35] A. Nagel, E. M. Stein, S. Wainger, Balls and metrics defined by vector fields. I. Basic properties, Acta Math. 155 (1985), no. 1-2, 103-147.

[36] S.D. Pauls,Minimal surfaces in the Heisenberg group. Geom. Dedic., (2004), 104, 201-231.

[37] M. Ritoré, C. Rosales, Area stationary surfaces in the Heisenberg group H1, Adv. Math. 219 no. 2 (2008) 633-671.

[38] L. P. Rothschild, E. M. Stein, Hypoelliptic differential operators and nilpotent groups, Acta Math. 137 (1976), no. 3-4, 247-320.

[39] F. Serra Cassano, D. Vittone, Graphs of bounded variation, existence and local boundedness of nonparametric minimal surfaces in Heisenberg groups, Adv. Calc. Var. 7 (2014), no. 4, 409-492.

[40] E. M. Stein, Harmonic analysis: real-variable methods, orthogonality, and oscillatory in- tegrals, Princeton Mathematical Series, vol. 43, Princeton University Press, Princeton, NJ, 1993, With the assistance of Timothy S. Murphy, Monographs in Harmonic Analysis, III.

[41] C.Y. Wang, Viscosity convex functions on Carnot groups, Proc. Amer. Math. Soc. 133 (2005), no. 4, $1247-1253$.

[42] C.Y. Wang, The Aronsson equation for absolute minimizers of $L^{\infty}$ functionals associated with vector fields satisfying Hörmander's condition. Trans. Amer. Math. Soc. 359 (2007), 91-113

Luca Capogna, Department of Mathematical Sciences, Worcester Polytechnic Institute, WORCESTER, MA 01609

E-mail address: lcapogna@wpi.edu

Giovanna Citti, Dipartimento di Matematica, Universitá di Bologna, Italy

E-mail address: giovanna.citti@unibo.it

Maria Manfredini, Dipartimento di Matematica, Universitá di Bologna, Italy

E-mail address: maria.manfredini@unibo.it 